\title{
THE EFFECT OF THE WEIGHTS LOADING ON THE FRONT OF FARM TRACTOR ON DEPTH STABILITY USING DISK PLOW
}

( Received: 27 .9. 2007)

\author{
By \\ A. M. A. Mamkagh \\ Plant Production Department, Faculty of Agriculture, Mu'tah University, Al-Karak, Jordan.
}

\begin{abstract}
The main experiment was conducted at the Agricultural Research Station of Mutah University , Jordan in 2005 to study the effect of different weights loading on the front of farm tractor $(0 \mathrm{~kg}, 100 \mathrm{~kg}$ and $200 \mathrm{~kg}$ ) and three plowing speeds $(5,7$ and $9 \mathrm{~km} / \mathrm{h})$ by $2 \mathrm{WD}$ and $4 \mathrm{WD}$ on depth stability using disk plow with $112 \mathrm{~cm}$ width. The plowing depth was adjusted at about $30 \mathrm{~cm}$.

The results showed that the plowing depth was significantly $(\mathrm{p}<0.05)$ affected by the weights loading on the front of the tractor, where as the plowing depth increased from $27.59 \mathrm{~cm}$ to $29.11 \mathrm{~cm}$ by loading $100 \mathrm{~kg}$, and from $27.59 \mathrm{~cm}$ to $28.52 \mathrm{~cm}$ by loading $200 \mathrm{~kg}$. But the plowing depth decreased from 28.84 $\mathrm{cm}$ to $27.23 \mathrm{~cm}$ by increasing the plowing speed to $9 \mathrm{~km} / \mathrm{h}$, and depth was significantly decreased by changing the wheel drive from $2 \mathrm{WD}$ to $4 \mathrm{WD}$.

The interaction between the loading weights, plowing speed and wheel drive showed that the depth of $31.17 \mathrm{~cm}$ was the more reliable to the adjusted depth during the experiments. This result was obtained from plowing speed about $7 \mathrm{~km} / \mathrm{h}$ and $2 \mathrm{WD}$ with $100 \mathrm{~kg}$ on the front of tractor which means more depth stability.
\end{abstract}

Key words: depth stability, disk plow , farm tractor, loading weights.

$$
\begin{aligned}
& \text { تأثير الوزن المضاف على مقدمة الجرار الزراعي على ثبات عمق الحراثة عند استخدام المحراث القرصي }
\end{aligned}
$$

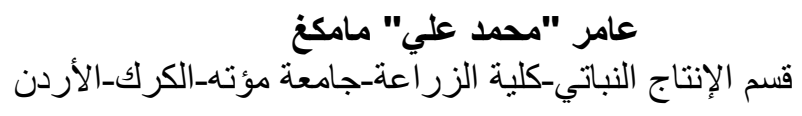

$$
\begin{aligned}
& \text { ملخص الزع }
\end{aligned}
$$

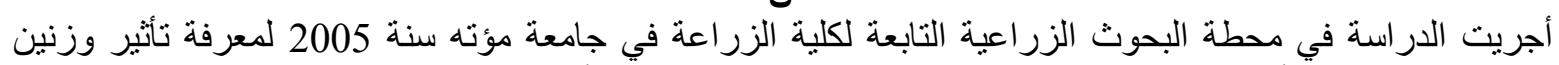

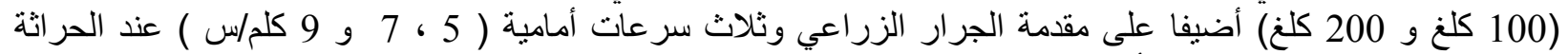

$$
\begin{aligned}
& \text { بمحر اث قرصي محمول ثنلاثي الأقر اص بعرض } 112 \text { سم على عمق } 30 \text { سم مع ثبات عمق الحر اثثة بالدفع الرباعي و الثنائي }
\end{aligned}
$$

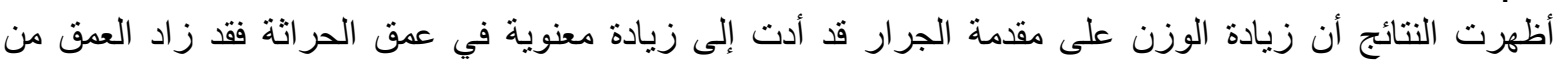

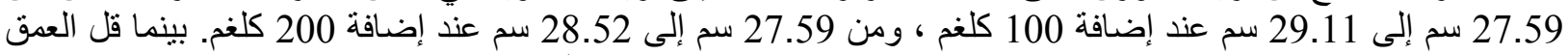

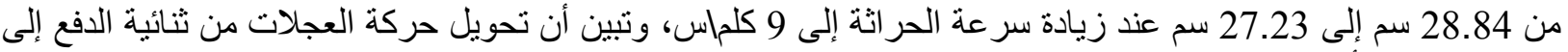

$$
\begin{aligned}
& \text { رباعية الدفع قد أدت إلى تقليل عمق الحر اثنة معنويا. }
\end{aligned}
$$

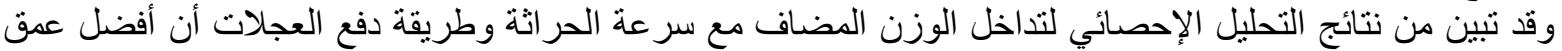

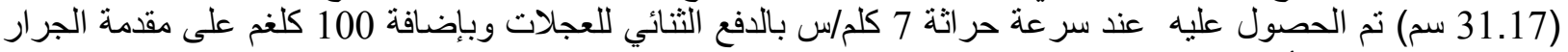

$$
\begin{aligned}
& \text { الزراعي مما يعني الأكثر انتظاما لعمق الحر اثنة. }
\end{aligned}
$$

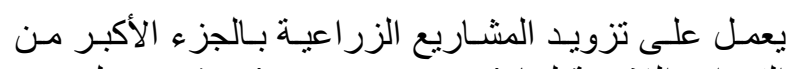

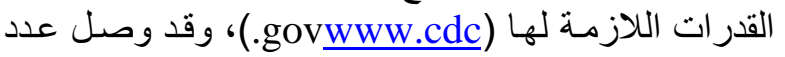

$$
\begin{aligned}
& \text { الجرارات الزراعية للعام } 2004 \text { في الوطن العربي وني إلى } \\
& 1
\end{aligned}
$$

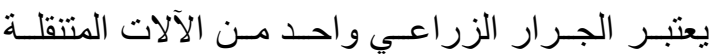

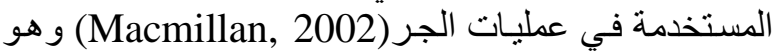


العجلات وطريقة شبك المحراث مع الجرار، فنسبة نوزيع

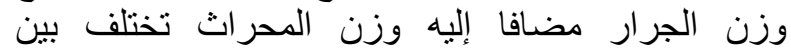
الجرار ات ثنائية الدفع ورباعية الدفع، وكذللك عند الحراثة الدئة

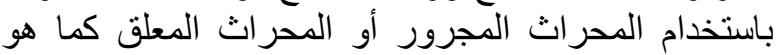
موضح في الجدول (1) (Taylor et al., 1991).

جدول (1): النسبة المئوية لتوزيع وزن الجرار والمحراث على المحور

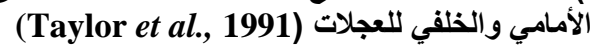

\begin{tabular}{|c|c|c|}
\hline المحور الخلفى & المحور الأمامي & الجر ار/المحر اث \\
\hline 65 & 35 & ثنائي الدفع/محمول \\
\hline 40 & 60 & رباعي الدفع/محمول \\
\hline
\end{tabular}

طرحت في السنوات الأخيرة عدة طرق للتنبؤ بأداء الجرار الزراعي على أساس مقدار الوزن التئ المضاف علئ عليه،

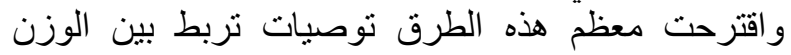

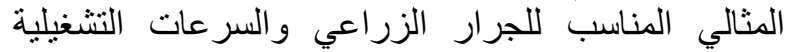
المختلفة ( Reece,1968; Brixius and Zoz,1976; الزعاعية Gee-Clough et al.1982; Brown,1982; Rutherford and McAllister,1983; Bloome et Domier و Hofman (1977) (al.,1983)، وقام كل من (1978)

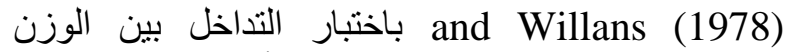
المضاف إلى العجلات و السرعة الأمامية للجية للجرارات

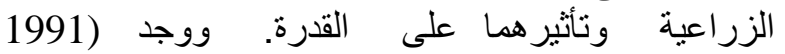
(Taylor et al .,

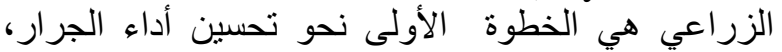

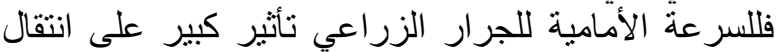

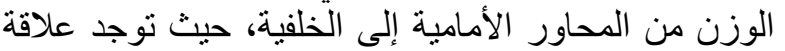
قوية بين سرعة الجرار والوالوزن الوزئ على العجلات القائدة فعند

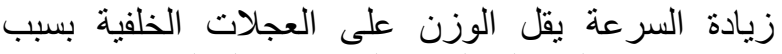

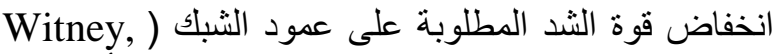

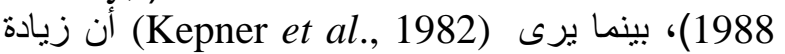

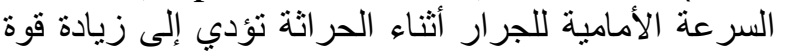

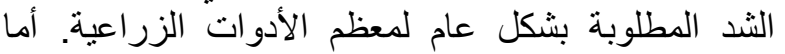

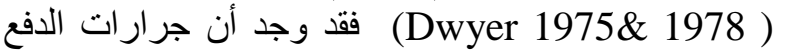
الثنائي لا تعطي قوة الثند القصوى بسبب السرعة البطيئة

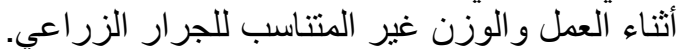

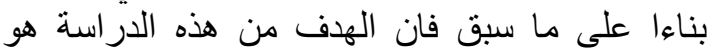

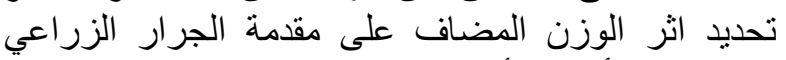
والسرعة الأمامية أثناء الحراثة عند الثتخديد الثرام المحراثي القرصي على ثبات عمق الحراثة الثنة وذللك بالدفع الرباعي و الثنائي لعجلات الجرار الزراعي.

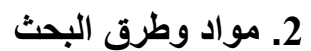

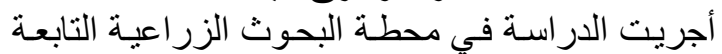

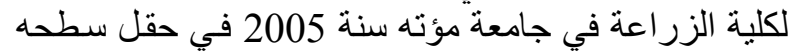

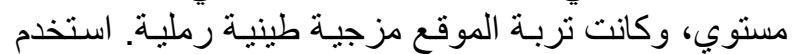

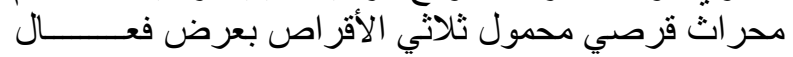

561464 جـر ار ، أمـا في المملكـة الأردنيـة الهاثتـمية فقدـ

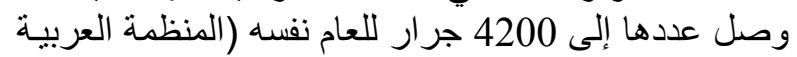

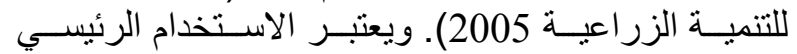

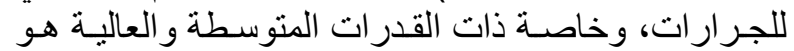

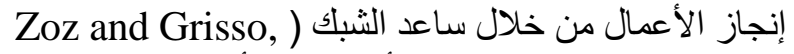

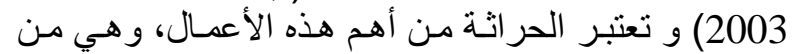

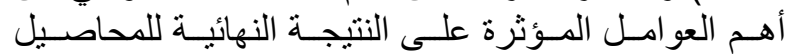
Thompson and Taylor, 1982; Varco et al., ) 1989; Pilbeam et al., 1991; De Costa et al.,

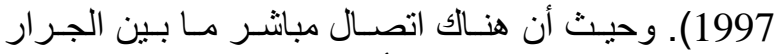

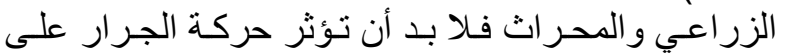

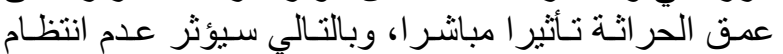

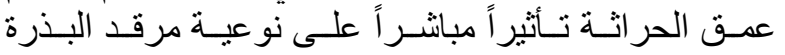

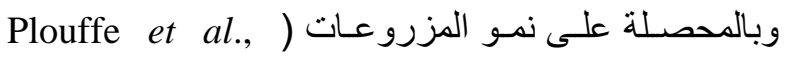
1995)، فنو عيـة مرقد البذرة عامل مهم لزيـادة المحاصيل

على سبيل المثال الحولية منها (Sing et al., 1994). لتجهيز مرقد البذرة بدايةً تستخدم أدوات الحئة الحراثة

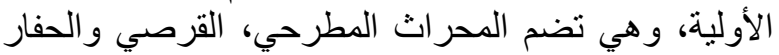

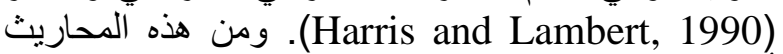

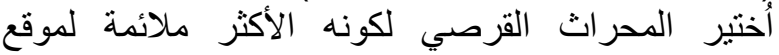

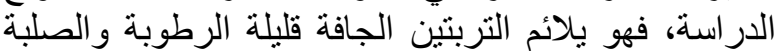

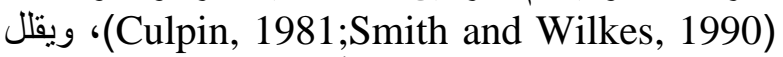
دن الاحتكاك من خلال دوران أقراصه بدلا من الانزلاق

داخل الأخدود (Harris and Lambert, 1990).

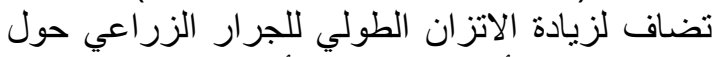

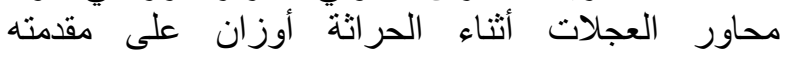

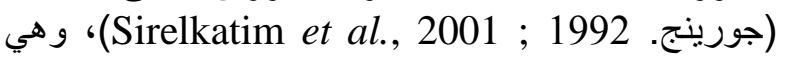
عبارة عن قطع حديدية كالمبينة في الثنكل (1)،

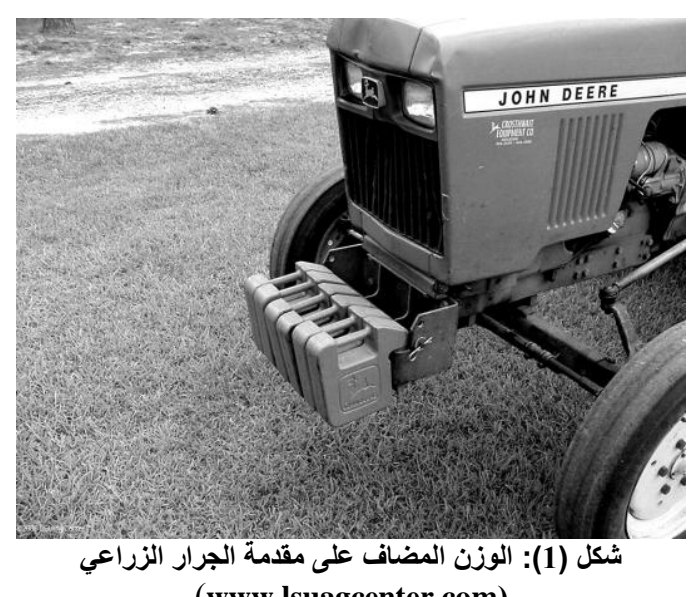

.(www.lsuagcenter.com)

فزيادة هذه الأوزان على مقدمة الجرار سوف تحد من انتقال

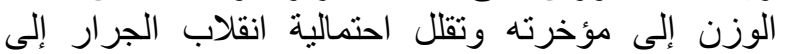
الخلف (Www.lsuagcenter.com) (Witney, 1988).

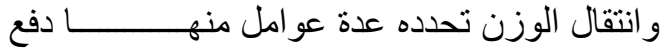


عمق الحر اثثة، فعند زيادة سرعة الحر اثتة إلى وكلماس قل العمق من 28.84 سم إلى زئى 27.23 سم، ويتضح ذلانك أيضا

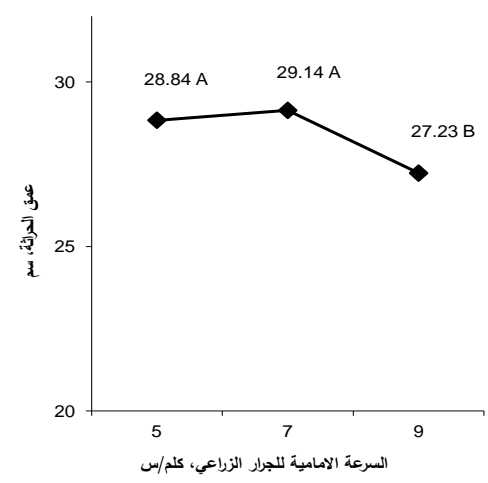

شكل (3): تأثير السرعة الأمامية للجرار على عمق الحراثة

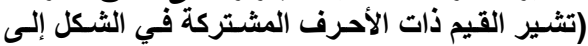

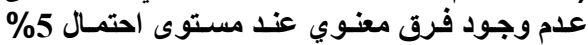

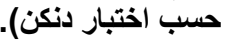

من خـلال الثـكل (4) الذي يبين تـأثير التـداخل بين

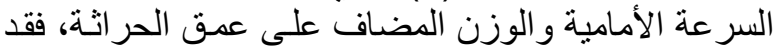

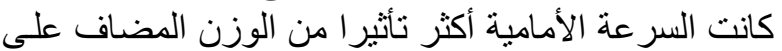

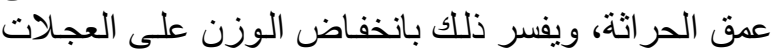

الخلفية عند زيادة السر عة الأمامية (Witney, 1988).

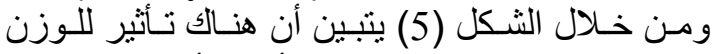

المضاف على عمق الحر اثثة، ولكن التأثير الأكبر كان

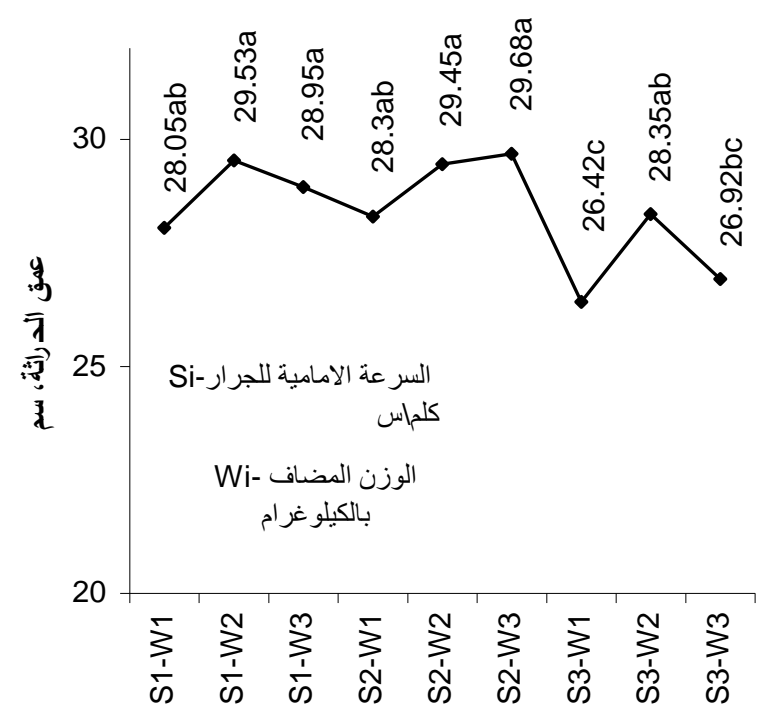

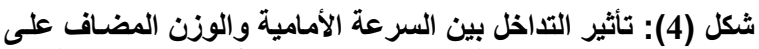

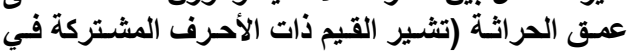

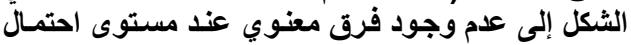
5 5 حسب اختبار دنكن).

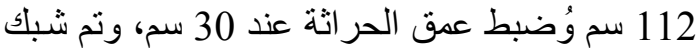

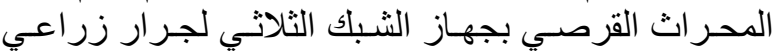

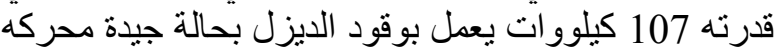

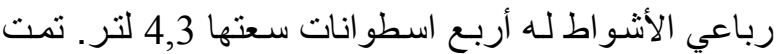

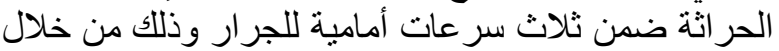

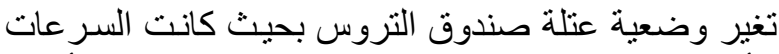

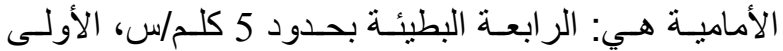

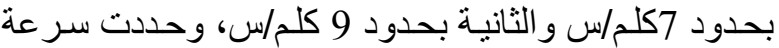

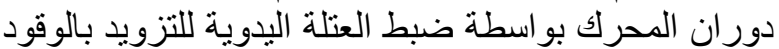

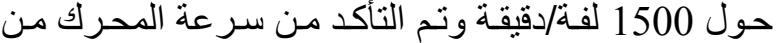
خلال مر اقبة المؤشر الخاص بذاصلك.

صمدت التجربة ضمن القطاعات العشوائية الكاملة

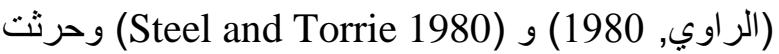
التربة عشو ائيا بدون أوزان على مقدمة الجرار، ثُ بـ بإضافة

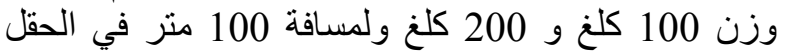
بثلاثة مكررات و أثناء ذللك تم تحويل الحركة من من التجاتهات

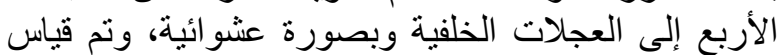
عمق الحر اثة كل 0.5 منر من كل مكرر.

\section{3. - 3. النتائج و المناقثة}

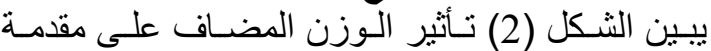

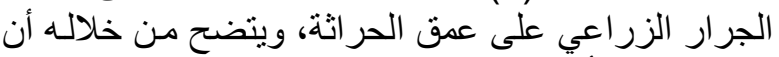

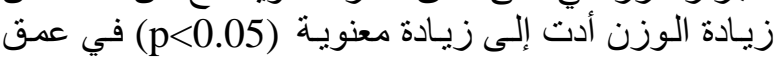
الحر اثة فقد زاد العمق من 27.59 سم إلى إلى 29.11 سم عند عند

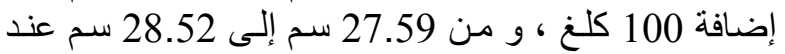

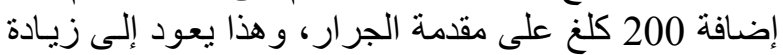

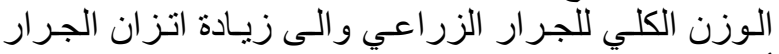
Sirelkatim et al., أثناء العمل(Witney, 1988

.(2001

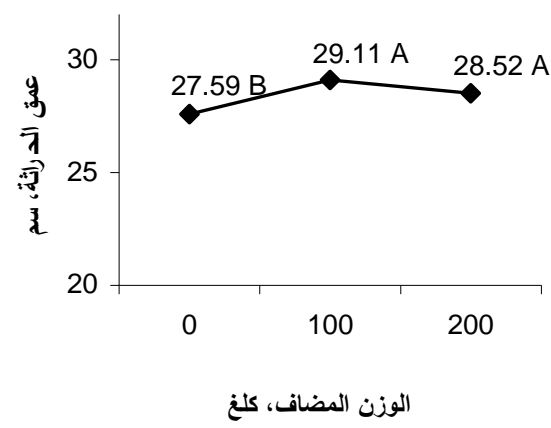

شكل (2): تأثير الوزن المضاف على عمق الحراثة. (تثبير

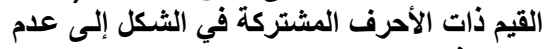

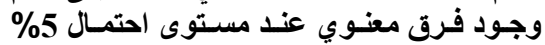
حسب (ختبار دنكن).

يوضــح الثـكل (3) تـأثير السـر عة الأماميـة للجـرار

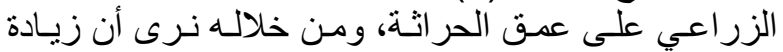
السرعة الأمامية للجرار أثناء الحر اثثة قد أثرت معنويـا على نلى الثي 
بالنظر إلى نتائج الدراسة نستنتج أن زيادة مقدار

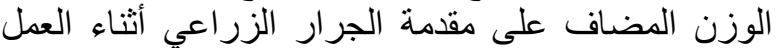

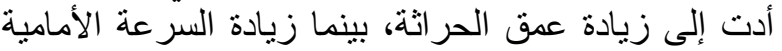

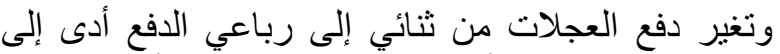

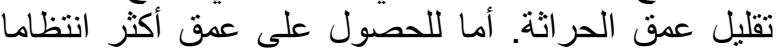

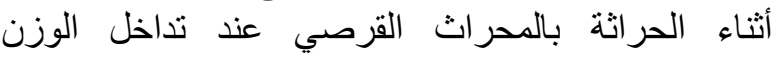
المضاف مع سرعة الحراثة وطريقة دفع العجلات وفي الثراء الثرني

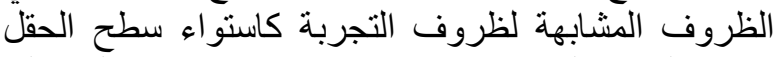
ونوع التربة المحروثة، نوصي بإضافة 100 كلغ كلغ على لئى

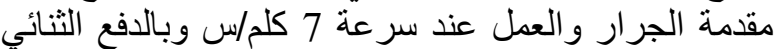
للعجلات مما يقلل أيضا من استهلاك الوقود أثناء العمل.

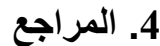

الراوي، خاشع محمود، خلف الله، عبد العزيز (1980).

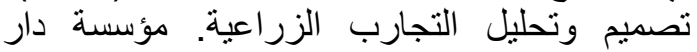

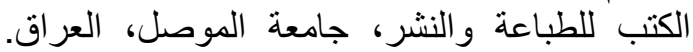

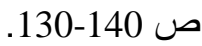

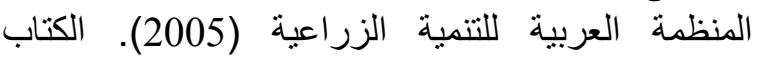
السنوي للإحصاءات الزراعية العربية. المجلد 25.

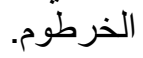
جورينج، كارول أي. (1992). قدرة المحرك والجرار.

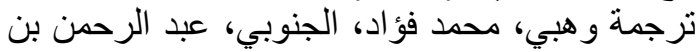

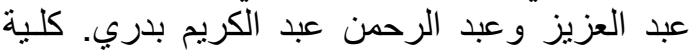

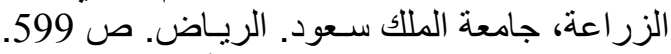

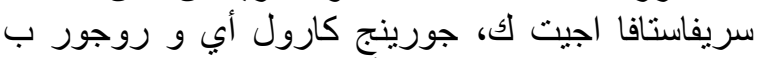

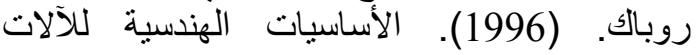
الزراعية. نرجمة السحيياني صالح عبد الرحمن، الريات

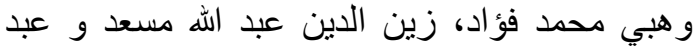

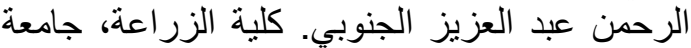

$$
\text { الملك سعود. الرياض. صئ. ص الريز } 264 .
$$

A guide to safe farm tractor operation. http://www.cdc.gov/nasa/ docs/ d0015 01d001600/d001534/d001534.html

Ballasting compact utility tractors. http://www. lsuagcenter.com/en/lawn_garden/homegardening/ mequipment/compact_tractors Ballasting+Compact+Utility+ ractors.htm Bloome P.D., Summers J.D., Khalinian A. and Batchelder. D.G. (1983). Ballasting recommendation for two wheel and four
بسـبب دفـع العجـاتل، فعند تحويـل الـدفع مـن ثنـائي إلـى

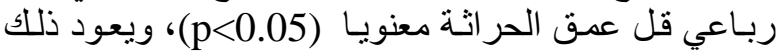

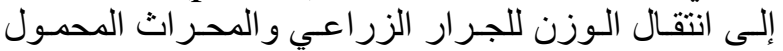

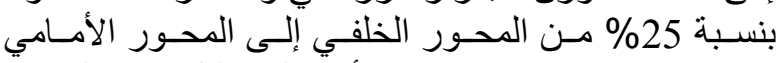

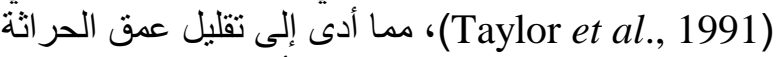

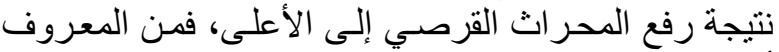

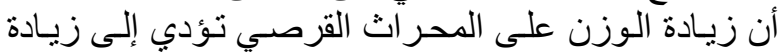

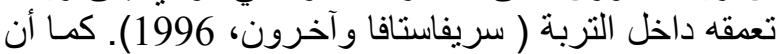
تقليل عمق الحراثة في هذه الحالة نتج عن عدم الاستخلال

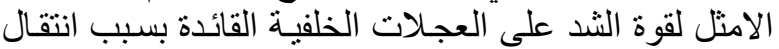

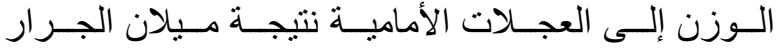

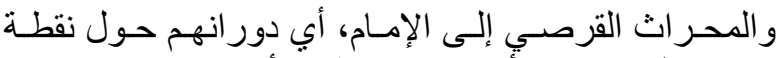

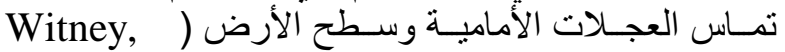
.)(1988

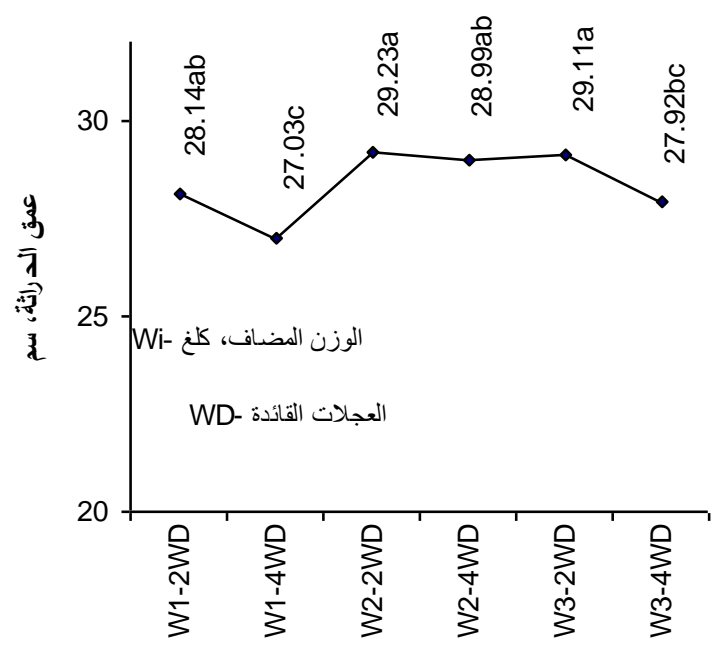

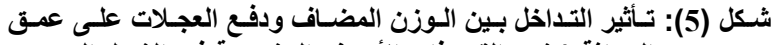

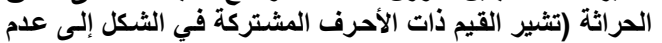

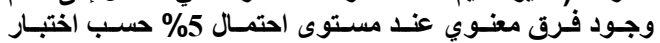
دنكن).

وقد تبين من نتائج التحليل الإحصائي لتداخل الوزن

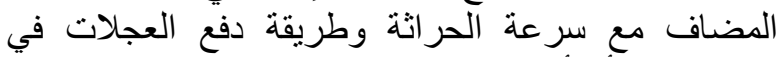

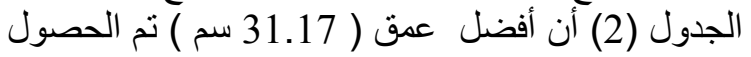

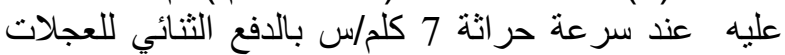

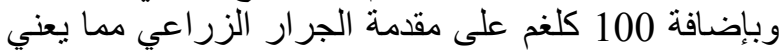
انتظاما لعمق الحر اثنة.

جدول (2): تأثير التداخل بين الوزن المضاف على مقدمة الجرار وسرعته الأمامية ودفع العجلات على عمق الحراثة.

\begin{tabular}{|c|c|c|c|c|}
\hline \multicolumn{3}{|c|}{ السرعة الامامية للجرار ،كلم/س } & \multirow[t]{2}{*}{ دفع العجلات } & \multirow{2}{*}{ على مقدمة الجراركلغ } \\
\hline 9 & 7 & 5 & & \\
\hline $25.50 \mathrm{~g}$ & $30.10 \mathrm{abc}$ & 28.83 bcde & ثنائى & \multirow[t]{2}{*}{ صفر } \\
\hline $27.33 \mathrm{efg}$ & $26.50 \mathrm{fg}$ & 27.27 efg & رباعى & \\
\hline 28.83 def & $31.17 \mathrm{a}$ & 28.70 bcde & ثنائي & \multirow[t]{2}{*}{100} \\
\hline 28.87 bcde & 27.73 def & $30.37 \mathrm{ab}$ & رباعى & \\
\hline 27.33 efg & $30.33 \mathrm{ab}$ & 29.67 bcde & ثنائى & \multirow[t]{2}{*}{200} \\
\hline $26.50 \mathrm{fg}$ & 29.03 bcde & 28.23 cdef & رباعى & \\
\hline
\end{tabular}


wheel drive tractors. ASAE, paper No.831067.St. Joseph, MI., USA.

Brixius W.W. and Zoz F.M. (1976). Tires and tracks in agriculture. American Society of Automotive Engineers, paper No. 760653, presented at Off-Highway Vehicle Meeting, Milwaukee, USA.

Brown G.A. (1982). Tractor Ballasting Recommendations. Conference on Agricultural Engineering, Armidale.

Culpin C. (1981). Farm Machinery. Tenth edition. Granada Publishing. London. p 76.

De Costa W.A. J.M., Dennet M.D., Ratnaweera U. and Nyalemegbe, K. (1997). Effects of different water regimes on field-grown determinate and indeterminate faba bean (Vicia faba L.). I, Canopy growth and biomass production. Field Crop Research. 49:.83-93.

Domier K.W. and Willans A.E. (1978). Tractive Efficiency-maximum or optimum. Translocation . of ASAE. 21(4): 650-659.

Dwyer M.J. (1975). Some Aspects of Tire Design and their Effect on Agricultural Tractor Performance. Proc. Inst. Mech. Engr. Conf. Off-Highway Vehicles. Tractors and Equipment. Institute of Mechanical Engineering, London.

Dwyer M.J. (1978). Maximizing Agricultural Tractor Performance by Matching Weight, Tire Size and Speed to the Power Available. Proc. $6^{\text {th }}$ Int. Conf. Soc. Terrain Vehicle System, Vienna.

Gee-Clough D., McAllister M. and Pearson G. (1982). Ballasting wheeled tractors to achieve maximum power output in frictional-cohesive soils. J. Agric. Engnr Res. 27: 1-19.

Harris P.S. and Lambert H.W. (1990). Farm Machinery and Equipment. TATA. McGrawhill Publishing Company Ltd. New Delhi. p. 114.

Hofman V.L. (1977). Model tractor demonstration on ballasting 2WD tractors and 4WD tractors for efficient use of horsepower. ASAE. Paper. No 77-151.

Kepner R.A., Bainer R. and Barger E.L. (1982). Principles of Farm Machinery. $3^{r d}$ Ed., p. 147149. The AVI Pub.Co.Inc. Westport.USA.

Macmillan R.H. (2002). The mechanics of tractor implement performance. http://www.eprints. unimelb. edu. au.
Pilbeam C.J., Hebblethwaite P.D., Nyongesa T. E. and Ricketts, H. E. (1991). Effects of autumn sowing dates on growth and yield of determinate and indeterminate field beans (Vicia faba L.). J. Agric. Sci. 116: 385-393.

Plouffe C., McLaughlin N.B., Tessier S. and Lague C. (1995) Energy requirements and depth stability of two different moldboard plow bottoms in a heavy clay soil. Canadian Agricultural Engineering. Canada 37(4): 279-285.

Reece A.R. (1968). Two-or four-wheel drive. Farm Machine Design Eng. 2(2).

Rutherford, I. and McAllister M. (1983). Tyres and Traction. (Advisory leaflet) ADAS.

Sing B., Chanasyk D.S., McGill W.B. and Nyborg M.P.K. (1994). Residue and tillage management effects on soil properties of a typic Cryoboroll under continuous barley. Soil and Till. Res. 32:117-133.

Sirelkatim K.A., Hasan A.A. and Mohamed O.S. (2001). The effect of some operating parameters on field performance of a 2WD tractor. Scientific Journal of King Faisal University (Basic and Applied Sciences).2(1): 153-166.

Smith H.P. and Wilkes L.H. (1990). Farm Machinery and Equipment. $6^{\text {th }}$ Ed. p. 120. TATA, McGraw-Hill Pub. Co. LTD. New Delhi.

Steel R. and Torrie J. (1980). Principles and Procedures of Statistics. A Biometrical Approach. $2^{\text {nd }}$ ed. Book-Mart Press, Inc. USA.

Taylor R., Schrock M. and Wertz K. (1991). Getting the most from your tractor. Farm Machinery and equipment. http://www.oznet.ksu.edu/library/ ageng2/mf588.pdf

Thompson R. and Taylor H. (1982). Prospects for Vicia faba L., in Northern Europe. Outlook Agric. 11: 127-133.

Varco J.J., Frye W.W., Smith M.S. and MacKown C.T. (1989). Tillage effects on nitrogen recovery by corn from a nitrogen-15 labelled legume cover crop. Soil Sci. Soc. Am. J. 53: 822-827.

Witney B. (1988). Choosing and Using Farm Machines. Longman Scientific and technical. John Wiley and Sons, Inc., New York. USA, pp.277-285.

Zoz F.M. and Grisso R.D. (2003). Traction and Tractor Performance. Agricultural Equipment Technology Conference, 9-11 February, Louisville, Kentucky USA. ASAE Publication Number 913C0403, p.1-47. 